\title{
Experimental leprosy in monkeys. I. Sooty mangabey monkeys: transmission, susceptibility, clinical and pathological findings
}

\author{
B. J. GORMUS*, K. XU, G. B. BASKIN, \\ L. N. MARTIN, R. P. BOHM, J. L. BLANCHARD, \\ P. A. MACK, M. S. RATTERREE, \\ H. M. MCCLURE $\dagger$, W. M. MEYERS $\ddagger \&$ \\ G. P. WALSH $\S$ \\ *Departments of Microbiology, Pathology and Veterinary Sciences, \\ Tulane Regional Primate Research Center (TRPRC), Covington, \\ LA, USA; †Yerkes Regional Primate Research Center, Emory \\ University, Atlanta, GA, USA; $\ddagger$ Armed Forces Institute of Pathol- \\ ogy, Washington, DC, USA and §Gerald P. Walsh, American \\ Leprosy Foundation, Rockville, MD, USA
}

Accepted for publication 30 January 1995

\begin{abstract}
Summary A total of 31 sooty mangabey monkeys (SMM) (Cercocebus torquatus at ys) inoculated by various routes with differing numbers of SMM-origin Mycobacterium leprae (ML) and 4 SMM inoculated with human-origin ML were observed for 4-12 years. SMM-origin ML was more pathogenic in SMM than human-origin ML. The spectrum of disease ranged from indeterminate to borderline and lepromatous in different animals. Some animals developed pure neural leprosy. Erythema nodosum leprosum (SNL) was also observed.

Combined intravenous/intracutaneous (IV/IC) routes of inoculation more effectively induced advancing, disseminated lepromatous forms of leprosy; IV or IC routes alone were less effective at comparable doses. Total IV/IC doses of SMM-origin ML equal to or greater than $5 \times 10^{8}$, with morphologic indices (MIs) ranging from 5 to $10 \%$, produced advancing, disseminated LL leprosy in $92 \%$ of SMM. Lower IV/IC doses and inoculations by a single IV or IC route produced fewer leprosy infections and more spontaneous regressions. As a species, captive SMM are highly susceptible to experimental leprosy and provide an excellent model for the longitudinal study of leprosy.
\end{abstract}

\section{Introduction}

We previously reported detailed descriptions of naturally-acquired leprosy in 2 wildcaught, captive sooty mangabey monkeys (SMM) ${ }^{1,2}$ The first, animal, A015, was diagnosed in 1979 and the second, G932, a former cagemate of A015, in 1986. The causative agent, identified unequivocally as Mycobacterium leprae (ML), was isolated 
Table 1. M. leprae exposure of miscellaneous SMM

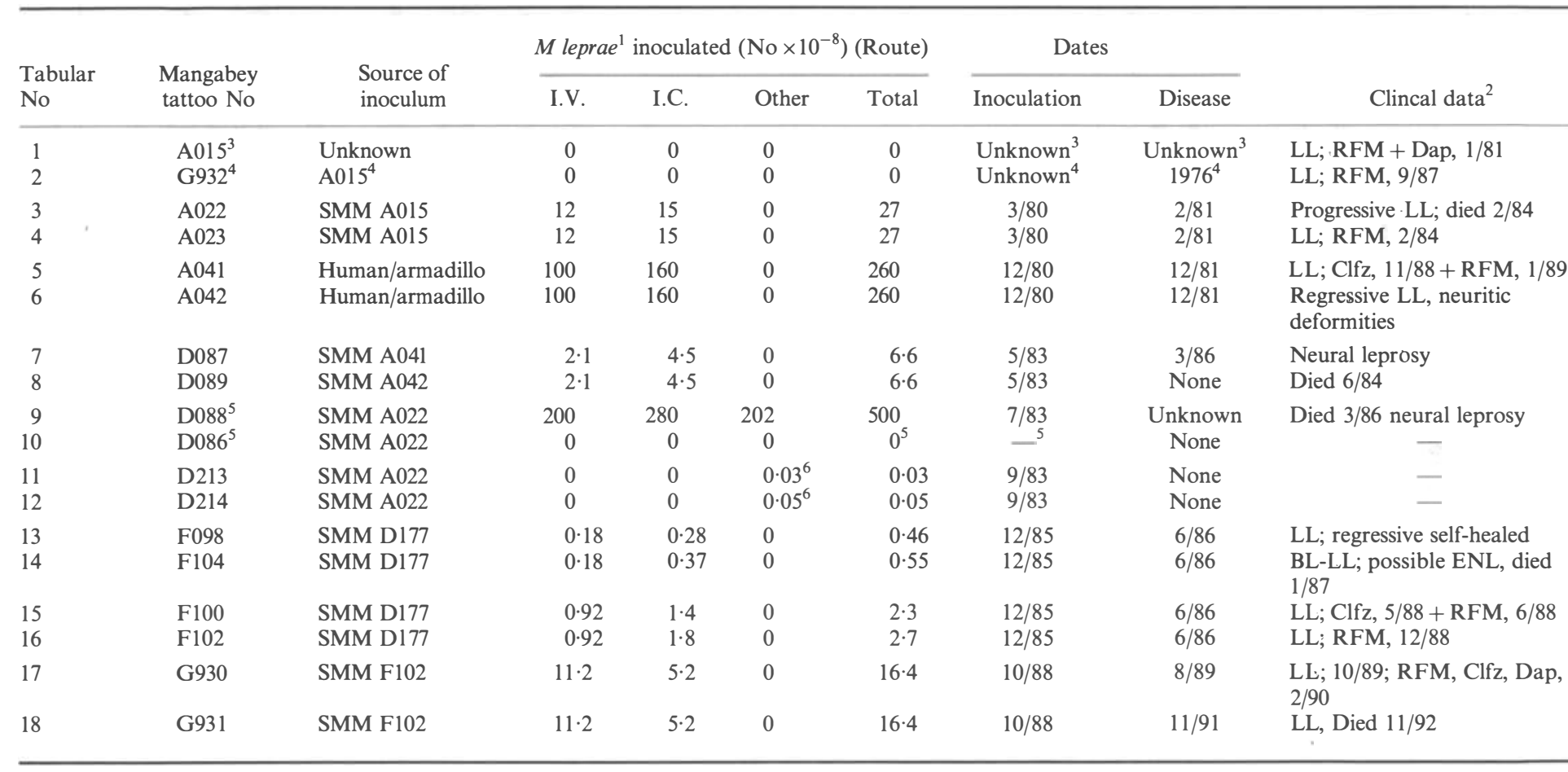

${ }^{1}$ MIs from 5 to $10 \%$.

${ }^{2} \mathrm{RMF}$, rifampicin; Clfz, clofazimine; Dap, dapsone.

${ }^{3}$ A 015 contracted leprosy by natural, unknown means, and had transient followed by sustained lesions for an unknown number of years before documentation of leprosy by biopsy in 1979 .

${ }^{4}$ G932 was housed for several years with A015 prior to 1979, and appears to have contracted leprosy from A015; leprosy was diagnosed in G932 in 1986 (Ref. ${ }^{1}$ ).

${ }^{5}$ D088 was inoculated by i.v. + i.c. + an intranasal route and remained housed together with its infant, D086.

${ }^{6}$ D213 and D214 were inoculated by exposure to aerosol-borne ML. 
Table 2. Inoculation ${ }^{1}$ of SMM with titrated $M$. leprae doses

\begin{tabular}{|c|c|c|c|c|c|c|}
\hline \multirow{2}{*}{$\begin{array}{l}\text { Tabular } \\
\text { No }\end{array}$} & \multirow{2}{*}{$\begin{array}{c}\text { Mangabey } \\
\text { tatoo No }\end{array}$} & \multicolumn{3}{|c|}{ M. leprae inoculated $\left(\right.$ No $\left.\times 10^{-8}\right)$ (Route) } & \multirow{2}{*}{$\begin{array}{c}\text { Disease } \\
\text { onset } \\
\text { (date) }\end{array}$} & \multirow[b]{2}{*}{ Clinical data } \\
\hline & & i.v. & i.c. & Total & & \\
\hline 19 & D171 & 200 & 280 & 480 & 9.83 & $\begin{array}{l}\mathrm{LL} ; \mathrm{RFM}^{2}, 1986 \text { regressive } \mathrm{LL} \text {, } \\
\text { neuropathy, }\end{array}$ \\
\hline 20 & D172 & 200 & 280 & 480 & $9 / 83$ & $\mathrm{CLFZ}^{2}, \mathrm{RFM}, 1988$ \\
\hline 21 & D173 & 20 & $28 \cdot 0$ & 48 & $7 / 91$ & $\begin{array}{l}\text { BL-LL neuropathy LL, } \\
\text { neuropathy, RFM }\end{array}$ \\
\hline 22 & D174 & 20 & $28 \cdot 0$ & 48 & $7 / 88$ & CLFZ, $7 / 88$ \\
\hline 23 & D175 & 2 & $2 \cdot 8$ & 4.8 & None & - \\
\hline 24 & D176 & 2 & $2 \cdot 8$ & $4 \cdot 8$ & $9 / 83$ & LL, RFM, 6/86 \\
\hline 25 & D177 & $0 \cdot 2$ & 0.28 & $0 \cdot 48$ & $9 / 85$ & LLs, neuropathy, RFM, 1/84 \\
\hline 26 & D178 & $0 \cdot 2$ & $0 \cdot 28$ & 0.48 & $6 / 86$ & neuroedema, neuropathy \\
\hline
\end{tabular}

\footnotetext{
${ }^{1}$ Inoculated in July, 1983; ML inoculum from SMM A022 (see No 11, Table 1); MI, 10\%.

${ }^{2}$ RFM, rifampin; Clfz, clofazimine.
}

Table 3. M. leprae inoculation ${ }^{1}$ of SMM: dose and route study

\begin{tabular}{|c|c|c|c|c|c|c|}
\hline \multirow{2}{*}{$\begin{array}{l}\text { Tabular } \\
\text { No }\end{array}$} & \multirow{2}{*}{$\begin{array}{c}\text { Mangabey } \\
\text { tatoo No }\end{array}$} & \multicolumn{3}{|c|}{ M. leprae inoculated $\left(\mathrm{No} \times 10^{-8}\right)($ Route $)$} & \multirow{2}{*}{$\begin{array}{l}\text { Disease } \\
\text { onset } \\
\text { (date) }\end{array}$} & \multirow[b]{2}{*}{ Clinical data } \\
\hline & & i.v. & i.c. & Total & & \\
\hline 27 & E045 & 4.5 & 6.2 & $10 \cdot 7$ & $6 / 84$ & $\begin{array}{l}\text { LL; disseminated; } \text { RFM }^{2} 11 / 85 \text {; } \\
\text { healed }\end{array}$ \\
\hline 28 & E042 & $4 \cdot 5$ & $5 \cdot 9$ & $10 \cdot 4$ & None & - \\
\hline 29 & E043 & $4 \cdot 5$ & $6 \cdot 2$ & $10 \cdot 5$ & $12 / 85$ & $\begin{array}{l}\text { LL; disseminated; neuropathy } \\
\text { involvement; RFM 5/87 }\end{array}$ \\
\hline 30 & E044 & $4 \cdot 5$ & $6 \cdot 0$ & $10 \cdot 5$ & $6 / 87$ & $\begin{array}{l}\text { LL; disseminated; died } 5 / 88 \\
\text { after RFM initiation }\end{array}$ \\
\hline 31 & E038 & $0 \cdot 04$ & $6 \cdot 0$ & $6 \cdot 0$ & $12 / 85$ & $\begin{array}{l}\text { Regressive/Progressive LL; } \\
\text { neuritic deformities; } \text { MCLN }^{2} \text {, } \\
1991\end{array}$ \\
\hline 32 & E039 & 0 & $6 \cdot 0$ & $6 \cdot 0$ & $6 / 84$ & $\begin{array}{l}\text { Regressive LL; self-healed } \\
\text { by } 1 / 88\end{array}$ \\
\hline 33 & D215 & 0 & $6 \cdot 0$ & $6 \cdot 0$ & None & - \\
\hline 34 & E040 & $4 \cdot 5$ & 0.06 & $4 \cdot 6$ & $10 / 84$ & $\begin{array}{l}\text { Disseminated/regressive; } \\
\mathrm{Clfz}^{2} ; 3 / 88+\mathrm{RMF}, 5 / 88\end{array}$ \\
\hline 35 & E047 & $4 \cdot 5$ & $0 \cdot 06$ & $4 \cdot 6$ & $4 / 85$ & $\begin{array}{l}\text { LL; disseminated; neuropathic } \\
\text { deformities; died } 1 / 88 \text { after } \\
\text { RFM initiation }\end{array}$ \\
\hline 36 & E041 & $4 \cdot 5$ & 0 & $4 \cdot 5$ & $5 / 84$ & Scrotal lesions, regressed \\
\hline 37 & E046 & $4 \cdot 5$ & 0 & $4 \cdot 5$ & $6 / 86$ & $\begin{array}{l}\text { Disseminated BL-LL; } \\
\text { neuropathic deformities; } \\
\text { RFM, } 5 / 87\end{array}$ \\
\hline
\end{tabular}

\footnotetext{
${ }^{1}$ Inoculated in February 1984; ML inoculum from SMM A022 (see No 11, Table 1); MI, $3 \%$.

${ }^{2}$ RFM, rifampin; Clfz, clofazimine; MCLN, minocycline.
} 
from A015 and used to inoculate additional SMM; ML from human sources was also utilized to inoculate SMM. ${ }^{3,4}$ These studies showed that SMM were susceptible to infection by ML from SMM or human sources. Starting in 1980, 31 SMM were inoculated with or exposed to ML originally isolated from SMM A015, and 4 SMM received human-origin ML. We summarize here our observations on these 35 experimentally- and the 2 naturally-infected SMM.

\section{Materials and methods}

Inoculations: in initial passage studies, nonulcerated lepromatous nodules were surgically harvested from A015; subsequently, subinoculated SMM were employed as a source of MI. Lepromata were obtained aseptically, minced and homogenized in cold phosphate-buffered saline using a Dounce homogenizer with a 40 ML mortar and Teflon pestle (Wheaton Scientific, Millville, NJ), passed through gauze and centrifiuged at $200 \times \mathbf{G}$ for $5 \mathrm{~min}$ at $4^{\circ} \mathrm{C}$. The acid-fast bacilli (AFB) in the supernatant were counted and morphologic indices (MI) determined by the method of Shepard \& McCrae. ${ }^{5}$ SMM were inoculated with ML suspensions, usually by combined intracutaneous (IC) and intravenous (IV) routes, using 2 IC sites per ear, the tip of the nose, an outer forearm, an outer calf and sometimes the periorbital regions. IV inoculations were made via the saphenous vein. In some experiments, titrated doses of ML were given by both routes, sometimes only a single route was utilized and, in a few instances, other routes of inoculation were used as described in the text.

Clinical observations: animals were observed daily and examined in detail 3-4 times per year or more, depending on the status of the animal, and the clinical aspects of the disease recorded. The Ridley-Jopling system was used to classify leprosy histopathologically. ${ }^{6}$

\section{Results}

Data describing 37 SMM, including the 2 natural cases, are presented in Tables $1-3$. Each SMM in the Tables is identified by the tattoo number and/or a tubular number to facilitate locating the animals to which reference is made in the text. The tabular numbers have no significance other than to aid in the discussion, but are arranged in chronological order with regard to the time of inoculation, in so far as possible. In all, 22 (Nos 3, 4, 9, 13-22, 24-27, 29-31, 34 and 35) of 24 (92\%) SMM given ML (MI = 5$10 \%$ ) of SMM-origin by both IV and IC routes developed leprosy within 2 months to 7 years postinoculation (PI) (SMM numbers 5-8 received human/armadillo-origin ML directly or after passage in SMMs A041 or A042, and were not included in the calculation of results with the SMM-origin inocula). D175 (No 23, Table 2) and E042 (No 28, Table 3) are the exceptions, showing no signs of leprosy by 121 months and 114 months PI, respectively.

Aerosol inoculation failed to produce leprosy in 2 SMM (D213 and D214, Nos 11 and 12, Table 1) and a third SMM, D088 (No 9), given IV + IC + intranasal inocula $\left(5 \times 10^{10}\right.$ total ML) initially developed necrotic lesions at IC inoculation sites followed by complete healing of visible cutaneous leprosy; following the death of D088 due to 
unknown causes, only neural lesions, classified as BB-BL with ENL, were identified histopathologically. ${ }^{7}$ An uninoculated infant, D086 (No 10), remained housed with its inoculated mother, D088, for 35 months PI without the baby developing leprosy. These latter SMM (Nos 9-12) were also not included in the susceptibility calculation.

A041 and A042 (Nos 5 and 6), (both SMM) were inoculated IV and IC with $2.6 \times 10^{10}$ total human ML passaged in an armadillo, and developed LL leprosy (Table 1). Although local lesions at inoculation sites developed within 1 year in both recipients of human/armadillo ML, they were slow to develop disseminated leprosy, and both had multiple episodes of spontaneous regression even though they were inoculated with the extremely large dose of greater than $10^{10} \mathrm{ML}$. The dermal disease in A042 regressed virtually completely within 5 years PI. There were, however, neuritic deformities, including an unusual involvement of the trigeminal nerve paralysing the muscles of mastication. Approximately 7 years were required for A041 to develop LL leprosy as advanced and as disseminated as that seen by $12-18$ months in most SMM inoculated with fewer SMM-origin ML (see Tables 1-3), and 2 SMM, D087 and D089 (Nos 7 and 8, Table 1), subinoculated with human-origin ML reisolated from A041 and A042, respectively, developed primary neuritic leprosy (D087) or remained asymptomatic 13 months PI when D089 died of unknown causes.

To examine the effect of dose, SMM D171-D178 (Nos 19-26) received titrated doses of SMM-origin ML by combined IV/IC routes (Table 2). All but D175 developed leprosy. The results from this group suggest that doses were effective down to almost $5 \times 10^{7}$ SMM-origin ML by the IV/IC route. This inoculum had an MI of $10 \%$; therefore, the minimal effective solidly staining dose tested is near $5 \times 10^{6}$. From this dose study, a relationship was previously suggested between susceptibility to leprosy and anti-PGL-I and anti-LAM IgG and IgM serum antibody responses. ${ }^{4,7,8}$ High levels of anti-PGL-I IgM antibodies correlated with a high susceptibility to multibacillary forms of leprosy and high anti-PGL-I IgG levels correlated with a resistance to multibacillary leprosy; high initial levels of anti-LAM IgG correlated with a higher probability of developing multibacillary leprosy. ${ }^{4,7,8}$

A second group of $11 \mathrm{SMM}$ were also given titrated doses of SMM-origin ML for further evaluation of the findings regarding dose effects in SMM D171-D178; groups receiving only IV or only IC inoculation were also included in this study (Table 3). Relationships between serum antibody responses to PGL-1 and LAM and susceptibility to leprosy among these 11 SMM are reported in Part II of this study. ${ }^{9}$

Among these $11 \mathrm{SMM}$, there was a dose relationship overall (Table 3) in that 3 of 4 high-total-dose SMM (E045, No 27, E043, No 29 and E044, No 30) developed progressive LL leprosy compared to 1 (E047, No 35) of 7 that developed progressive LL leprosy from lower total doses. Considering all forms of persisting leprosy, 3 (E045, No 27; E043, No 29 and E044, No 30) of 4 high-dose, 1 (E038, No 31) of 3 intermediatedose and 3 (E040, No 34; E047, No 35 and E046, No 37) of 4 low-dose recipients developed disease (Table 3). Lower-dose recipients had more regressive leprosy episodes and more disease nearer the borderline to borderline lepromatous (BB-BL) or subpolar lepromatous forms and more neural disease (Table 3). There was also a route effect in that, of the 7 SMM with persisting leprosy, 6 received ML by combined IV/IC routes. Only E046, No 37, developed persisting BL-LL leprosy with neuropathy after inoculation by a single (IV) route, and 2 SMM inoculated by only an IC route (E039, No 32 and D215, No 33) were leprosy resistant (Table 3). 
In this study, 3 of 4 SMM (E045, E043 and E044) given a total of more than $1 \times 10^{9}$ ML by combined IV/IC routes developed advancing, disseminated LL leprosy within 4-40 months and ultimately required treatment (Table 3). These 3 SMM fell into a common grouping designated susceptible. The fourth of these SMM (E042) failed to develop disease, and was grouped together with other SMM (see below) designated resistant.

E045 (No 27, Table 3) had advanced LL leprosy at inoculation sites and at sites distant from inoculation (dissemination) by 17 months PI. E045 was highly susceptible to leprosy requiring chemotherapy at 21 months PI.

E043 (No 29, Table 3) developed LL leprosy at or near inoculation sites and disseminated sites including the scrotum, but was prone to extreme enlargement of peripheral nerves, especially the superifical peroneal, radial and ulnar with associated neuropathic deformities. E043 was designated susceptible.

In all, $1(\mathrm{E} 047,35)$ of $3 \mathrm{SMM}$ (No 31, 34 and 35) inoculated with $4.56-6.04 \times 10^{8}$ total ML by combined IV/IC routes developed disseminated, advancing LL leprosy with neuritic deformities and died 48 months PI after the initiation of rifampicin (RFM) chemotherapy (Table 3). E047 (No 35, Table 3) was highly susceptible to disseminated LL leprosy and was grouped with the susceptible SMM.

Of the other 2 SMM (E038 and E040, Nos 31 and 34) receiving 4.56-6.04 $\times 10^{8} \mathrm{ML}$ by IV/IC routes, E038 became positive for AFB in nasal secretions by 10 months PI and developed progressive BB-BL leprosy at IC inoculation sites by 22 months PI followed by a period of spontaneous regression to the point of apparent inactivity (progressive/ regressive leprosy). Leprosy re-emerged in E038 26 months PI, was shown to be borderline (BB) leprosy at 76 months PI and lesions again self-healed by 83 months PI. Lesions re-emerged 90 months PI as subpolar lepromatous leprosy $\left(\mathrm{LL}_{\mathrm{s}}\right)$ with dissemination to uninoculated sites (progression) and neuritic deformities.

The history of disease in SMM E038 can be summarized as progressive/regressive/ progressive/regressive/progressive. E038 suffered multiple neuropathic deformities, presumably as a result of the multiple episodes of self-healing. E038 was placed on minocycline (MCLN) monotherapy 7 years PI; therapeutic response was dramatic with diminishing numbers of solidly-staining AFB in the tissues and healing of all lesions within 4-6 weeks.

E040, the other recipient of $4 \cdot 56-6 \cdot 04 \times 10^{8} \mathrm{MI}$ by combined IV/IC routes, developed indeterminate (Ind) leprosy within 8 months PI. By 26 months PI, ulcerated lesions were seen on uninoculated digits of the feet. These lesions were not biopsied. By 32 months PI, a dermal nerve in a lower leg was enlarged to $3 \mathrm{~mm}$ wide and dermal lesions appeared to heal. By 46 months PI, AFB-positive nasal smears were obtained, LL leprosy was observed at inoculation sites (No 34, Table 3) and dissemination was confirmed by lesions appearing on the uninoculated tail. This was followed by spontaneous regression of dermal lesions and the appearance of neuritic deformities. After 49 months, continued exacerbation of some lesions was noted, classifiable as subpolar lepromatous leprosy $\left(\mathrm{LL}_{\mathrm{s}}\right)$ with extensive neuritic involvement which, by 51 months PI, became classifiable as primary neural $\mathrm{LL}_{\mathrm{s}}$ leprosy requiring treatment to halt the progress of neuropathic deformities. At 50-51 months, some biopsies of E040 lesions showed Ind leprosy and biopsies from other lesions showed primary neural $\mathrm{LL}_{\mathrm{s}}$ leprosy. E040 had widespread evidence of bone reabsorption and atrophy (several digits were reabsorbed and approximately $50 \%$ of the tail was removed due to bone absorption and self-mutilation). 
E038 and E040 fell into a common grouping designated susceptible with resistance to indicate that they developed persisting leprosy with periods of regression of symptoms followed by periods of continued progression, and/or initially developed indeterminate (Ind) (E040) or BB-BL leprosy (E038). E040 subsequently developed $\mathrm{LL}_{\mathrm{s}}$ dermally and neurally and E038 subsequently developed $\mathrm{BB}$, then $\mathrm{BB}-\mathrm{BL}$ and, eventually $\mathrm{LL}_{\mathrm{s}}$ leprosy. E040, therefore, appeared to be more susceptible (less resistant) than E038, based on clinical observations and on the BB classification, since BB-forms of leprosy have more cell-mediated immunity $(\mathrm{CM})$ (and more resistance) against ML than LLforms. $^{6}$

Of 2 SMM given $4.5 \times 10^{8}$ ML by the single IC route, E039 developed LL leprosy limited to inoculation sites within 4 months PI (Table 3), but these self-healed within 41 months PI and failed to reappear; the other (D215) failed to show symptoms of leprosy to date (118 months PI). E039 and D215 were grouped as resistant SMM.

Of 2 SMM (E041 and E046) given $4.5 \times 10^{8}$ ML by IV route alone, E041 (No 36) developed, within 3 months, erythematous lesions at the base of and on the skin and foreskin of the penis and on and near the scrotum, indicative of disseminated leprosy. These spontaneously regressed without recurrence of any disease (grouped as resistant); E046 (No 37) developed disseminated, progressive/regressive BL-LL leprosy by 24 months PI with continued progression of symptoms by 30 months PI when a biopsy indicated BL leprosy (Table 3). E046 developed severe neuropathic deformities in addition to significant dermal manifestations and required chemotherapy by 39 months PI. E046 was grouped with the susceptible SMMs because of the BL-LL characteristics of the disease and because of the relatively rapid progression of disease.

To summarize the results of the dose/route study, E045 (No 27), E043 (No 29), E044 (No 30), E047 (No 35) and E046 (No 37) were classified as susceptible; E038 (No 31) and E040 (No 34) as marginally susceptible or susceptible with resistance; and E042 (No 28), E041 (No 36), D215 (No 33) and E039 (No 32) as resistant.

\section{Discussion}

Susceptibility to experimental leprosy varied among individual SMM inoculated on the same day with identical IV and IC doses of ML from a given batch of SMM-origin inoculum. Doses of SMM-origin ML of $1 \times 10^{9}$ or greater by combined IV/IC routes produced LL leprosy in 11 out of 12 recipients (A022, A023, G930, G931, D171, D172, D173, D174, E045, E043 and E044, Tables 1 and 2). The time of disease onset varied from 2 to 96 months among these 11 SMM. Of these 11 SMM, D172 had self-regressive LL leprosy although he was a high-dose recipient. D172 was ultimately treated with clofazimine (CLFZ) and RFM because of severe neuritic complications. The regressive nature of the LL leprosy in D172 suggests that he was a leprosy-resistant animal. It is tempting to speculate that such an animal might not have developed disease or might have developed leprosy closer to the TT end of the spectrum if he had been inoculated with a lower dose of ML. A 12 th SMM that received $1 \times 10^{9}$ SMM-origin ML by combined IV/IC routes, SMM E042 (No 28, Table 2) has shown no signs of leprosy to date (110 months PI).

In $8 \mathrm{SMM}$ receiving SMM-origin ML at lower doses of $2 \cdot 3-6 \times 10^{8}$ by IV/IC routes, no disease was produced in D175 (No 23) within 110 months PI; progressive LL leprosy 
was observed in F100 (No 15), F102 (No 16), D176 (No 24), E046 (No 37) and E047 (No 35); progressive $\mathrm{BB}-\mathrm{BL}$ or $\mathrm{BL}-\mathrm{LL}$ forms with regressive episodes occurred in E038 (No 31) and E040 (No 34), respectively (Tables 1 and 2).

Doses of SMM-origin ML lower than $1 \times 10^{8}$ by combined IV/IC routes produced self-healing, regressive LL leprosy in F098 (No 13), BL-LL leprosy with ENL in F104 (No 14), LL $\mathrm{s}_{\mathrm{s}}$ with neuropathy in D177 (No 25) and gross nerve enlargement/neuropathy in D178 (No 26) (Table 1).

Among 4 SMM given 4.5-6 $\times 10^{8}$ SMM-origin ML by a single IV or IC route, only 1 animal (E046, No 37) developed progressive, disseminated BB-BL ${ }^{6}$ leprosy (Table 2). The other 3 SMM (D215 (No 33), E039 (No 32) and E041 (No 36)) given $4.5 \times 10^{8}$ ML by a single route either failed to develop or sustain progressive leprosy. In this experiment (Table 3), 6 out of 7 SMM given $4 \cdot 5-10 \cdot 7 \times 10^{8}$ ML by combined routes (including 3 (E038, E040 and E047) that received all but $4-6 \times 10^{4}$ ML by a single route) developed sustained, progressive leprosy compared to only 1 (E046) out of 4 with sustained disease after inoculation by a single route. Thus, either the IV or IC route alone appears to be less effective than the combined routes in inducing progressive LL leprosy at a given dose. Even when the vast majority of inocula is given by only 1 route together with $40,000-60,000 \mathrm{ML}$ by the other route, this combined IV/IC regimen appears more effective than a single IV or IC route alone.

These findings suggest that the susceptibility of SMM to leprosy depends upon variable individual animal factors and is dependent on the dose and route of inoculation. The exact factors responsible for this variability are not known but may be partly genetic or immunologic in origin.

The pathogenicity of the ML from different sources appears to vary. SMM-origin ML is highly pathogenic for SMM, but human-origin ML passaged through an armadillo was much less effective in inducing progressive LL leprosy in 2 SMM even with large doses $\left(2 \cdot 6 \times 10^{10} \mathrm{ML}\right)$ of inocula (A041, No 13 and A042, No 14, Table 1), and 2 additional SMM subinoculated with $6.6 \times 10^{8}$ human-origin $\mathrm{ML}$ reisolated from SMM's A041 and A042 also developed disease more slowly or not at all (D087, No 7, and D089, No 8, Table 1). D089 died 13 months PI due to apparently unrelated causes with no signs of leprosy at necropsy; D087 developed $\mathrm{AFB}^{+}$nasal secretions 34 months PI with nerve enlargement by 79 months PI and a skin lesion classified as indeterminate leprosy 102 months PI. These findings suggest that human-origin ML are less pathogenic in SMMs than ML or SMM-origin. The reasons for this difference are not known. Possible strain differences of these ML were not investigated, but all microbiological and genetic parameters of these 2 strains investigated thus far appear similar. ${ }^{2}$ These ML isolates were not compared to each other in control mouse footpad studies to distinguish between strain variation as opposed to ML adaptation to the host species; unfortunately, the isolates are no longer available for study.

SMM D088 (No 9) was inoculated by IV/IC routes with $4.8 \times 10^{10} \mathrm{SMM}$-origin ML together with $2 \times 10^{9}$ by intranasal instillation (Table 1). No clinical disease was detected in D088 other than prominent regressive, necrotic lesions at all IC inoculation sites within weeks of inoculation. These later healed, but at death 35 months PI, there was widespread peripheral leprous neuritis, classified as pure neuritic leprosy in the BL-LL area of the spectrum. ${ }^{1-7}$ Intraneural ENL was also present in D088. ${ }^{1}$ An infant, D086 (No 10) housed together with its mother, D088, failed to show any signs of clinical leprosy, which may not be surprising since D088's disease was confined to the nerves. 
The possibility that the intranasal instillation of $\mathrm{ML}$ was responsible for a skin sensitization (vaccination?) resulting in the enormous reactions at dermal inoculation sites of D088 is plausible in view of the fact that 2 other SMM, D213 (No 11) and D214 (No 12), that were inoculated only by a n aerosol route (Table 1) have never developed clinical signs of leprosy and are strongly lepromin skin-test positive (B. Gormus, unpublished observations). Uninoculated normal SMM and those with advancing disseminated LL leprosy are usually lepromin-negative (manuscript in preparation). These intriguing suggestions require further investigation.

In conclusion, captive SMM are highly susceptible to leprosy and provide a useful model for the study of clinical leprosy. Much of the spectrum of leprosy was observed among the inoculated SMM, and many of the complications and sequelae observed in human leprosy patients were also manif est in these nonhuman primates. It is virtually impossible to study the natural history of leprosy early afterinf ection in humans, but the SMM model permits such an approach. The SMM model also permits the measurement of baseline, preinfection (control) data in individual animals and the longitudinal monitoring of each parameter in each animal with time in relation to individual baseline values and in relation to the progress of the disease.

\section{Acknowledgments}

This work was supported by NIH grant No AI 19302 from the National Institute of Allergy and Infectious Diseases and grant No RR 00164 from the Animal Resources Program, National Center for Research Resources. We thank Ms Ann Bennett for assistance with manuscript preparation and the following persons for expert technical assistance: Ms's Cynthia Trygg, Doris O'Leary and Phyllis LeBlanc.

\section{References}

1 Gormus BJ, Wolf RH, Baskin GB, Ohkawa S, Gerone PJ, Walsh GP, Meyers WM, Binford CH, Greer WE. A Second sooty mangabey monkey with naturally-acquired leprosy: first reported possible monkey-tomonkey transmission. Int J Lepr, 1988; 56: 450-7.

2 Meyers WM, Walsh GP, Brown HL, Binford CH, Imes GD, Jr., Hadfield TL, Schlagel CJ, Fukunishi Y, Gerone PJ, Wolf RH, Gormus BJ, Martin LM, Harboe M, Imaeda T. Leprosy in a mangabey monkeynaturally-acquired inf ection. Int $J$ Lepr, 1985; 53: 1-14.

3 Wolf RH, Gormus BJ, Martin LN, Baskin GB, Walsh GP, Meyers WM, Binford CH. Experimental leprosy in three species of monkeys. Science, 1985; 227: 529-31.

${ }^{4}$ Gormus BJ, Ohashi DK, Ohkawa S, Walsh GP, Meyers WM, Brennan PJ, Trygg C. Serologic responses to Mycobacterium leprae-specific phenolic glycolipid-I antigen in sooty mangabey monkeys with experimental leprosy. Int J Lepr, 1988; 56: 537-45.

5 Shepard CC, McCrae DH. A method for counting acid-fast bacteria. Int J Lepr, 1968; 36: 78-82.

6 Ridley RS, Jopling WH. Classification of leprosy according to immunity: a five-group system. Int J Lepr, 1966; 34: 255-273.

7 Baskin GB, Gormus BJ, Xu K, Martin LN, Wolf RH, Cantrell C, Pezeshkpour GH, Walsh GP, Malaty R, Meyers WM. Experimental borderline lepromatous leprosy with intraneural erythema nodosum leprosum in a mangabey monkey (Cercocebus atys). Int J Lepr, 1991; 59: 618-623.

8 Gormus BJ, Xu K, Meyers WM, Walsh GP, Levis WR, Meeker HC. Antibodies to lipoarabinomannan antigen in sooty mangabey monkeys experimentally inoculated with Mycobacterium leprae. Int J Lepr, 1990; 58: 65-72.

9 Gormus BJ, Xu K, Cho S-N, Baskin GB, Bohm RP, Martin LN, Blanchard JL, Mack PA, Ratterree MS, Meyers WM, Walsh GP. Experimental leprosy in monkeys. II. Longitudinal serological observations in sooty mangabey monkeys. Lepr Rev, 1995; 66: 105-25. 